There is no doubt that lysergic acid diethylamide or its metabolites are excreted in the bile. In experiments in which labelled diethylamide was injected into rats with bile fistulæ, Stoll et al. found that the bile contained within $2 \mathrm{hr}$. 70 per cent of the total radioactivity. The greater part of lysergic acid diethylamide undergoes chemical alteration because the excreted compounds or metabolites, in contrast to the diethylamide, were water-soluble. Paper chromatography revealed that the bile contained three radioactive compounds with the respective $R_{F}$ values of $0,0 \cdot 13,0 \cdot 18$. These compounds have not yet been identified chemically or biologically. The last two compounds give the Van Urk colour reaction and the blue fluorescence, characteristic for derivatives of lysergic acid, but neither of them is the 2-oxy-lysergic acid diethylamide, a compound obtained by Axelrod et al., with in vitro "enzyme systems present in mammalian liver microsomes when supplemented with oxygen and a reduced triphosphopyridine nucleotide generating system". The problem of whether the third metabolite found in bile, which no longer gives the Van Urk colour reaction, is identical with 2-oxy-lysergic acid diethylamide has still to be examined.

Sonnenweg 6,

\section{E. Rothlin}

Basle.

Oct. 25.

${ }^{1}$ Axelrod, J., Brady, R. O., Witkop, B., and Evarts, E. V., Nature, $178,143(1958)$

${ }^{2}$ Ianz, U, Cerletti, A., and Rothlin, E., Helv. Physiol. Acta, 13, 207 (1955).

a Boyd, E. S., Rothlin, E., Bonner, J. F., Slater, J. H., and Hodge, H. C., J. Pharmacol., 113, 6 (1955).

4 Stoll, A., Rothlin, E., Rutschmann, J., and Schalch, W. A., Experientia, i1, 396 (1955).

\section{Inhibition of Phosphoglucose Isomerase}

THE key carbohydrate intermediate, glucose 6-phosphate, which may follow several alternative metabolic pathways including the 6-phosphogluconate oxidative cycle and the glycolytic sequence, enters the latter route by the phosphoglucose isomerase reaction. This reaction, in which glucose 6-phosphate is reversibly converted into fructose 6-phosphate, could conceivably play a part in the regulation of carbohydrate metabolism, although it has seldom been considered from this point of view, doubtless because of the extremely high activity of the enzyme in most tissues and because the reaction has so far appeared to be very little affected by extraneous influences. The present evidence suggesting that the enzyme is strongly inhibited by 6-phosphogluconic acid, an intermediate in the direct oxidative pathway, may therefore be of interest in connexion with studies on the mechanism responsible for the partitioning of carbohydrate between the direct oxidative pathway and the glycolytic route.

The enzyme reactions were carried out by a technique similar to that described by Bodansky ${ }^{1}$. Crude phosphoglucose isomerase extracts from mammalian blood, liver and muscle were employed. Various preparations of 6-phosphogluconic acid made by the bromine oxidation of glucose 6-phosphat ${ }^{2}$ were used. With glucose 6-phosphate at a concentration of $0.002 M$ as the substrate, inhibitions of the order of 95 per cent were observed in the presence of equimolecular concentrations of 6-phosphogluconic acid, and inhibitions of about 75 per cent were obtained using $0.001 M$ 6-phosphogluconate. A similar inhibitory effect was demonstrated using fructose 6-phosphate as substrate.

Department of Biochemistry,

London Hospital Medical College, Turner Street,

London, E.I. July 19.

1 Bodansky, O., Cancer, 7, 1191 (1954).

¿ Robison, R., and King, E. J., Biochem. J., 25, 323 (1931). Seegmiller, J. E., and Horecker, B. L., J. Biol. Chem., 192, 175 (1951).

\section{Separation of Neovitamin $A_{1}$ from All-Trans Vitamin $A_{1}$ by Chromatography on Alumina}

IN fish liver oils and synthetic preparations of vitamin $A_{1}$ it is possible to estimate the contents of the two isomers, neovitamin $A_{1}$ and all-trans vitamin $A_{1}$, by spectrophotometry following separation by chromatography on activated secondary calcium phosphate (dicalcium phosphate), length of column 300-600 mm. '.

Even when the dicalcium phosphate has been subjected to the activation process previously described $^{1}$, the separation of the two vitamin $\mathrm{A}_{1}$ isomers obtained is not equally satisfactory on all commercially available dicalcium phosphate preparations.

Earlier investigations have shown that a poor separation of the two vitamin A isomers is obtained by chromatography on columns consisting of the comparatively coarse-grained alumina which is usually marketed as suitable for ehromatographic purposes. It has now been observed that good separation is obtained by chromatography on alumina prepared from fine-grained aluminium hydroxide.

The fractionation curve shown in Fig. 1 represents the results obtained by chromatographing $1.5 \mathrm{mgm}$. of a mixture of the alcohols of synthetic neovitamin $\mathbf{A}_{1}$ and all-trans vitamin $\mathbf{A}_{1}$. Chromatography was performed on a $75 \mathrm{~cm} . \times 0.9 \mathrm{~cm}$. column of aluminium oxide prepared as follows. Mixtures of diethyl ether and light petroleum with increasing contents of diethyl ether (2-25 per cent $v / v)$ were used for the elution. Eluate was collected in 4-ml. fractions. On the basis of measurements of $E_{325}$ for the individual fractions the ratio of the two vitamin $A_{1}$ isomers could be calculated. The result was : neovitamin : all-trans vitamin $=30: 70$.

The alumina used for the chromatography was prepared from aluminium hydroxide, analytical

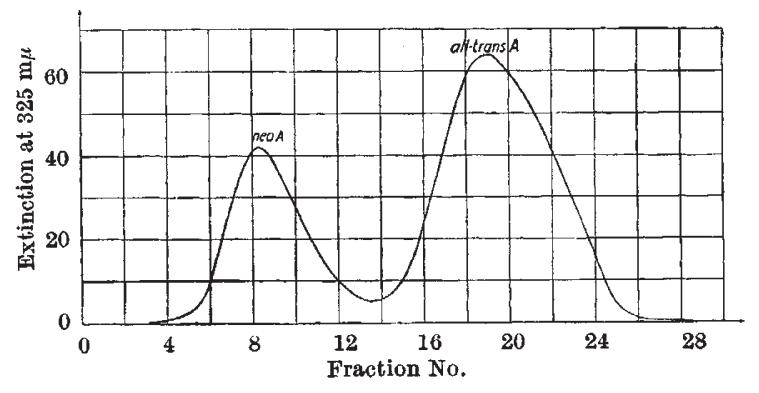

Fig. 1. Fractionation curve for synthetic neo- + all-trans-vitamin Fig. 1. Fractionation curve for synthetic neo- + all-trans-vitamin alumina 